\title{
Taxonomy of Algorithm Animation Languages
}

\author{
Ville Karavirta* \\ Department of Computer Science and Engineering \\ Helsinki University of Technology \\ Department of Computer Science and Engineering \\ Helsinki University of Technology \\ Lauri Malmi \\ Department of Computer Science and Engineering \\ Helsinki University of Technology
}

\begin{abstract}
In this paper, we present a taxonomy of algorithm animation languages, and apply it to evaluate a set of current languages. The taxonomy can be used by algorithm visualization system designers as a tool to compare visualization system languages with each other as well as for designing and implementing new systems and language features. In addition, the taxonomy provides guidelines to the features that are needed for transferring animations from one system to another. This is an ongoing project that supports the work started by the ITiCSE visualization working group in 2005 .
\end{abstract}

CR Categories: D.3.2 [Programming Languages]: Language Classifications-Specialized application languages

Keywords: software visualization, scripting languages

\section{Introduction}

Algorithm animation tools have been developed over two decades. The field has developed a variety of technical approaches and tools for both general and specialized use. Some tools operate on lowlevel graphical primitives and some others on high level concepts, thus reflecting the needs for which they have been originally developed. This variety makes comparison of tools somewhat complicated. Moreover, it complicates the issue of transforming animation information between systems, a feature that would be desirable for many users.

Definition of a general-purpose representation of animations was one of the goals of the ITiCSE working group in 2005 (Developing XML-based tools to support user interaction with algorithm visualization [Naps et al. 2005]). This is, however, not a trivial task due to the great variety of approaches in different visualization tools. Some systems (see, e.g., [Karavirta et al. 2004]) use an internal representation that is never shown to the user. Some have a scripting language intended to be written by hand or generated by a program and then animated by a system. This approach is utilized, for example, in JAWAA [Akingbade et al. 2003] and Animal [Rößling and Freisleben 2002].

\footnotetext{
*e-mail: vkaravir@cs.hut.fi

†e-mail: archie@cs.hut.fi

†e-mail: lma@cs.hut.fi

Copyright $\odot 2006$ by the Association for Computing Machinery, Inc.

Permission to make digital or hard copies of part or all of this work for personal or classroom use is granted without fee provided that copies are not made or distributed for commercial advantage and that copies bear this notice and the full citation on the first page. Copyrights for components of this work owned by others than ACM must be honored. Abstracting with credit is permitted. To copy otherwise, to republish, to post on servers, or to redistribute to lists, requires prior specific permission and/or a fee. Request permissions from Permissions Dept, ACM Inc., fax +1 (212) $869-0481$ or e-mail permissions@acm.org.

SOFTVIS 2006, Brighton, United Kingdom, September 04-05, 2006

(c) 2006 ACM 1-59593-464-2/06/0009 $\$ 5.00$
}

The working group concluded that by providing XML definitions of the essential elements of a visualization, the aspects of program dependency and tight coupling of the animation objects with the visualization software can be eliminated. The criteria for a common algorithm animation language specification are that it must be able to express the data structures and graphical primitives used in the visualization, hierarchies of animation operations, and various style settings for the data structures and graphical primitives. The working group specified six different elements intrinsic to visualization systems: objects (e.g., data structures) that are the focus of a visualization, graphical primitives (squares, circles, lines, etc.), transformations on graphical primitives (e.g., scaling, rotation), narration (text, graphics, audio), questions and feedback inserted in an animation, and metadata that describe the content of an animation.

Challenges, however, remain to be met. Existing systems need conversion modules for transforming animation information into and out of the system. Moreover, novel systems will be designed with their own internal representations that fit to the special needs of the system. In this paper, we wish to contribute to these issues by analyzing existing languages and approaches in order to find various ways to meet the criteria. We present a taxonomy for classifying algorithm animation language features. The taxonomy aids the visualization system designers to compare their designs with other systems and reflect on the needs of various features.

The rest of the paper is organized as follows. First, we report on the analysis done in Section 2. Section 3 in turn defines a taxonomy for categorizing and comparing the different algorithm animation language definitions. Finally, in Section 4 we make some conclusions.

\section{Data Description Languages}

The results of the literary survey [Karavirta 2005] conducted for this paper are summarized in the following subsections. First, we briefly demonstrate a number of algorithm animation languages used in existing algorithm animation systems. Second, we discuss some other languages surveyed that are related to or suitable for expressing algorithm animations. Several other systems and languages were studies in the literature survey as well, but are not covered in this paper. Excluded languages typically included similar features to the ones chosen for this paper with a wide variety of different syntaxes. On the other hand, taxonomies such as this can never be complete due to the new functionality constantly added to the languages; thus taxonomies are intrinsically supposed to be extended in the future. This is taken into account and we also mention some evident ways to extend the current taxonomy in parts that are too extensive to be completely covered here. For example, some animation languages include a complete programming language that could have been analyzed by a taxonomy of its own. 


\subsection{Algorithm Animation Languages}

Algorithm animation languages are tied to existing algorithm animation systems. They typically support both static (e.g., the state of the data structures) and dynamic visualizations (e.g., operations on the data structures). As an example, we will consider the case where a key is inserted into a binary search tree, and the search path during the insertion is highlighted. Figure 1 illustrates step by step such an animation. It should be noted that the script examples below do not comprise a complete animation, but are only fragments of the language.
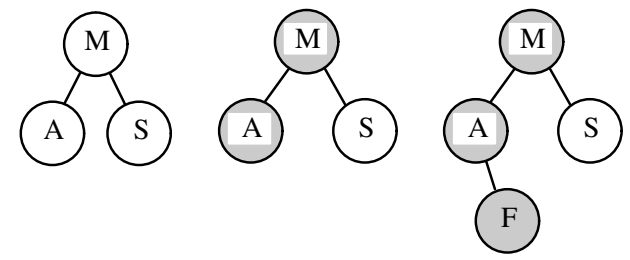

Figure 1: Pictures illustrating how the example animation used throughout the chapter could be visualized.

\subsubsection{AnimalScript}

ANIMALSCRIPT [Rößling and Freisleben 2000] is a scripting language for the ANIMAL [Rößling and Freisleben 2002] algorithm visualization system. ANIMALSCRIPT provides support for graphical primitives such as arc, circle, ellipse, line, point, polygon, polyline, square, and text. Coordinates for the objects can be given either in absolute coordinates or relative to another object or location. ANIMALSCRIPT supports also data structures array and list as well as code animation. Listing 1 shows how the example case can be described using ANIMALSCRIPT.

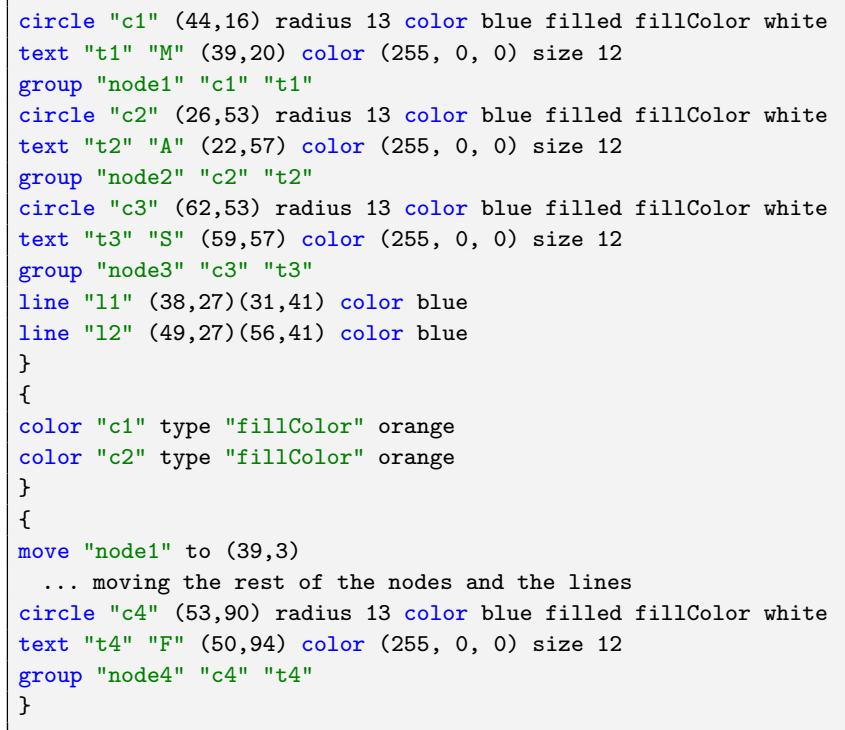

Listing 1: Example of graphical primitives and basic animation in ANIMALSCRIPT.

ANIMALSCRIPT2 [Rößling et al. 2004] introduces programming concepts including loops, conditionals, arithmetic expressions, and integer variables.

\subsubsection{DsCats}

DsCats [Cappos and Homer 2002] includes a command language for defining the animations. The language supports the definition of the structures, insert and delete operations, break points, annotations, and several options. An example of the BST insert is represented in Listing 2. As DsCats has automatic visualization, the highlighting of the search path cannot be done in the language. Furthermore, DsCats uses numbers instead of alphabets.

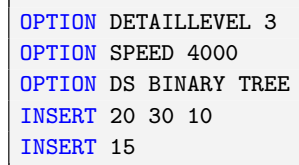

Listing 2: DsCats command language example.

\subsubsection{JAWAA}

JAWAA 2.0 [Akingbade et al. 2003] includes a scripting language for creating animations and viewing them over the web. JAWAA commands are divided in three types: primitive objects, action commands, and data structures.

Primitive objects in JAWAA are circle, rectangle, line, polygon, and oval. Action commands allow movements and changes to objects or object groups. Listing 3 shows an example using primitive objects.

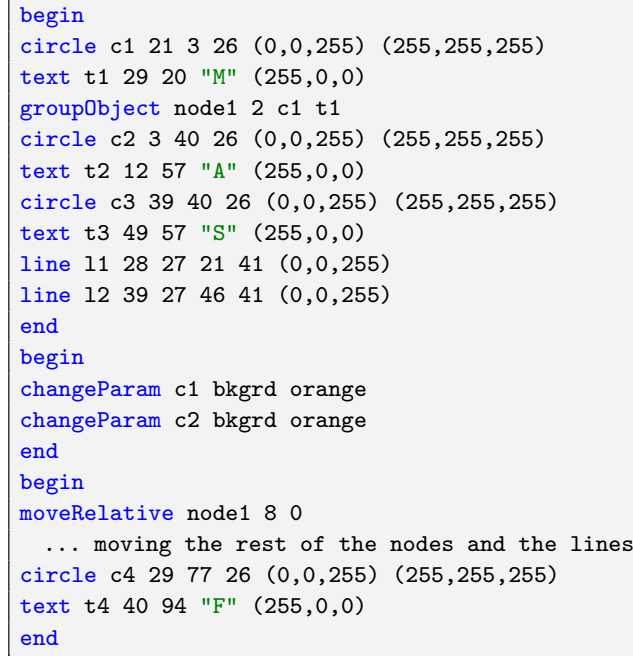

Listing 3: An example of JAWAA primitives and action commands.

Data structures specified in JAWAA language are array, queue, stack, list, tree, and graph. These structures have the normal operations, for example, stack has the operations push and pop and queue operations dequeue and enqueue. Listing 4 shows an example using data structures. However, according to documentation, trees do not work in the current version of JAWAA.

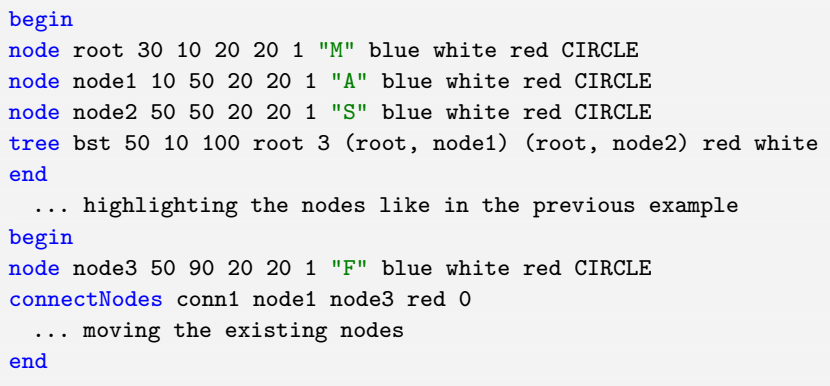

Listing 4: An example of JAWAA data structures and operations. 


\subsubsection{Matrix ASCII}

MatrixPro [Karavirta et al. 2004] includes a textual format for describing data structures. The language supports the definition of structures in three representations: edge list, adjacency list, and array. In addition, the layouts used for the structures, and some visual properties of the layouts can be defined. Listing 5 gives an example of this format. The example defines the same tree as in the previous examples. Matrix ASCII does not support animation, thus the insert and highlight operations are not included.

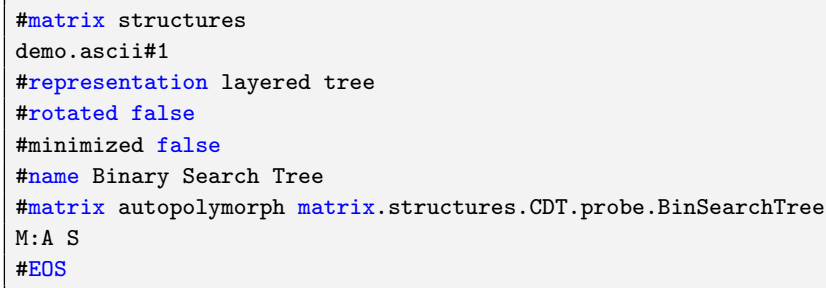

Listing 5: Matrix ASCII format example of a tree.

\subsubsection{SALSA}

SALSA (Spatial Algorithmic Language for Storyboarding) [Hundhausen and Douglas 2002] is a high-level language for creating low fidelity storyboards. SALSA enables layout and logic of visualization to be specified in terms of its spatiality among visual objects.

SALSA includes three data types: cutout, position, and s-struct (spatial structure). Cutout is a computer version of a paper cutout. Position represents a point in the coordinate space. A spatial structure is a region in which cutouts can be arranged according to a particular spatial layout pattern (for example, grid).

The 12 commands of SALSA can be divided in four categories: element creation and modification, conditional execution, iteration, and animation. As the approach of SALSA is completely different from the other languages, we do not show an example of the same use case as for the rest of the languages (although it could be done).

\subsubsection{Samba}

The two main categories of Samba [Stasko 1997] commands create graphical objects and modify these. Graphical objects available are line, rectangle, circle, triangle, polygon, and text. The modification commands include, for example, commands to move and exchange objects, to change the colors, and to change text and visibility of objects. Many of the modifications can be done either as smooth animation or in one frame (discreet change). Listing 6 gives an example of a Samba script.

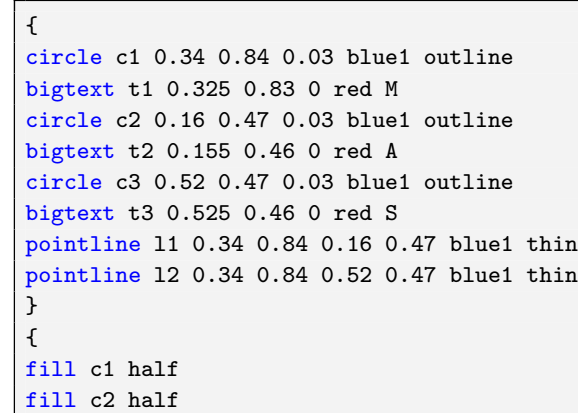

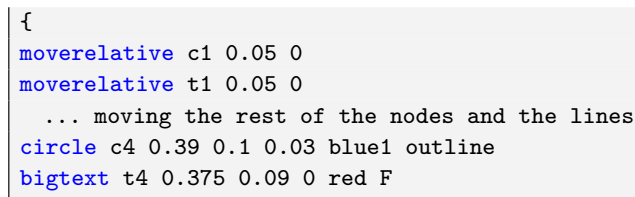

Listing 6: Example of Samba command language.

Samba also has a support for multiple views by introducing two commands that can be used to define a view and setting the current view. In addition, Samba has many front-ends, for example, JSAMBA [Stasko ] and JHAVÉ [Naps et al. 2000].

\subsection{Other Languages}

There exist several other languages related to algorithm animation. For example, graph description languages are relevant because many of the common data structures can be described as graphs. Examples of graph description languages are GraphXML [Herman and Marshall 2000] and GXL (Graph eXchange Language) [Winter 2001]. GraphXML is a graph description language designed to be used between graph drawing and visualization software. GXL is an XML language designed to be a standard format for data exchange between graph-based tools.

Since algorithm animations are often described using graphical primitives, also graphical description languages are relevant here. The following subsection introduces one such language, Scalable Vector Graphics (SVG) [W3C 2001].

\subsubsection{Scalable Vector Graphics (SVG)}

Scalable Vector Graphics (SVG) [W3C 2001] is an XML language targeted for describing graphics. The key features that we are interested in here are the graphical shapes defined in SVG. These are rectangle, circle, ellipse, line, polyline, and polygon. Another interesting feature of SVG is the animation. Listing 7 gives an example of using shapes and animation. Note, that the comments in the listing represent parts that have been left out of the example.

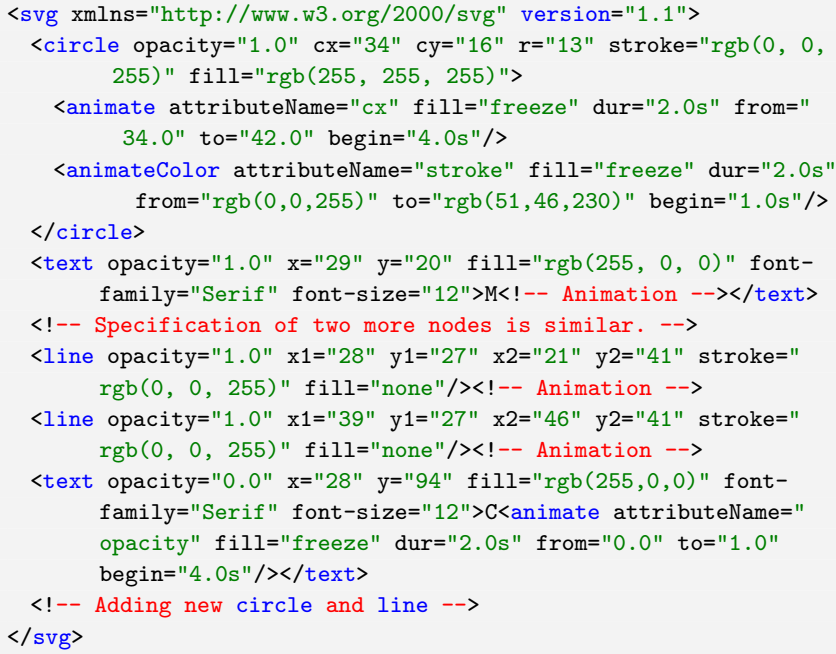

Listing 7: SVG shape and animation example. 


\section{Taxonomy}

Based on the survey of the algorithm animation languages in the previous section, we define a taxonomy of algorithm animation languages to evaluate the languages. The top-level of the taxonomy is represented in Figure 2

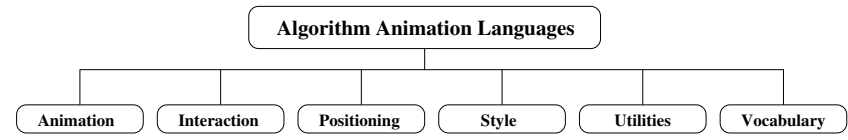

Figure 2: Taxonomy of Algorithm Animation Languages.

The main categories of the taxonomy are Animation, Interaction, Positioning, Style, Utilities, and Vocabulary. Animation describes the level and versatility of the animation capabilities of the language. Interaction describes the level and type of interaction provided for the end user of animations. Utilities inspects properties of the language not directly related to algorithm animation but still helpful in the process of creating them. Positioning considers the variety of different ways to position the objects, and Style the variety of styling facilities. Vocabulary describes the degree of supported object types, i.e. the building blocks used in the animation.

In the following sections, we will describe the categories of the taxonomy in more detail. In addition, we will evaluate the algorithm animation languages introduced in the previous section using the newly defined taxonomy. The reader should keep in mind that we evaluate the languages, not the systems: a feature might be available in a system, but not supported by the algorithm animation language. For example, MatrixPro has many of the features available in the GUI, but the ASCII format supports only a few of them. The internal representation and the possibility to save animations as serialized Java objects is not considered to be an animation language.

On the other hand, animation languages are not subsets of system features either. For example, Matrix ASCII includes support for commenting the textual description that is not a system feature nor accessed through the GUI in any way. Similar features emerging primarily from animation languages are metadata support, debug facilities, and some coordinate system issues. However, as many features in animation languages and animation systems do overlap, we have decided to adopt the used terminology from the Principled Taxonomy of Software Visualization by Price et al. [Price et al. 1993] where applicable. That work synthesizes some other well known taxonomies as well.

\subsection{Category Animation}

Description The category Animation (see Figure 3) describes the animation effects as well as how the final animation can be customized through the language. Animation has four subcategories: data structure (DS) operations, grouping, timing, and visual attribute animation.

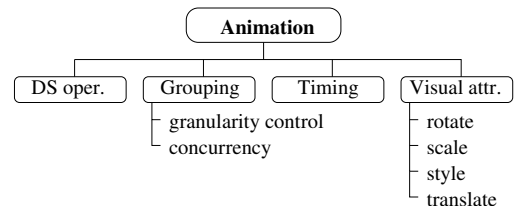

Figure 3: Category Animation.

Many algorithm animations are intended to visualize data structures such as trees and graphs and abstract data types (ADT) like stacks, queues, and dictionaries. The first subcategory - data structure operations - describes the functionality for data structures and ADTs, for example, the push operation for stacks and the connect nodes operation for graphs.

Grouping inspects the support for grouping the animation operations to be animated sequentially and/or simultaneously. Grouping has two subcategories granularity control, which states whether or not the sequential animation operations can be grouped to form smaller and bigger steps, and concurrency that determines whether the language supports definition of concurrent animations.

Timing examines the support for timing of the animation. Timing includes, for example, the possibility to set the duration and start time of an animation operation.

Visual attribute animation describes the visual animation techniques available in the language. This has four subcategories: rotate, scale, style, and translate. Rotate and scale states whether the objects can be rotated and scaled, respectively. Style describes the dynamic support for style attributes such as color, fill color, and line style. Translate examines how the position of an object can be changed.

Evaluation The languages are evaluated in category Animation in Table 1. As can be seen, the amount of different data structure operations varies greatly. Only DsCats and JAWAA support highlevel operations like insert, delete, push, and pop. Matrix ASCII has no support for animation at all

SVG has significantly more flexible timing features than the other languages. Actually, timing possibilities are not available at all in several other languages. However, in SVG, the animation operations can be set a minimum or maximum duration, number of repeats, repeat duration, key times, etc.

In the visual attribute animation subcategories, the systems can be roughly divided into two groups: those that support them and those that do not. The scaling of single objects is not supported in Samba. However, the whole view can be zoomed, thus the yes in parentheses. In SVG, almost all properties of the graphical objects can be animated, and only the most common are mentioned in the table.

\subsection{Category Interaction}

Description The category Interaction describes the type and level of interaction that can be specified using the language. Interaction has two subcategories: Control and Engagement (see Figure 4). Emphasis should be placed on distinguishing between the interaction provided by the tool and interaction supported by the language. This distinction is not always clear.

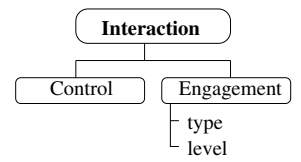

Figure 4: Category Interaction.

Control measures the ways the execution of the animation can be controlled from the language. Almost all of the current algorithm animation systems provide a control panel with play, rewind, speed, etc. options. However, we are interested in controlling them by the language. Examples of control are play, speed, stop, step forward, and step backward. 
Table 1: Evaluation of the languages in category Animation.

\begin{tabular}{|c|c|c|c|c|c|c|c|c|}
\hline \multirow[t]{2}{*}{ Language } & \multirow[t]{2}{*}{ DS operations } & \multicolumn{2}{|c|}{ Grouping } & \multirow[t]{2}{*}{ Timing } & \multicolumn{4}{|c|}{ Visual attribute animation } \\
\hline & & 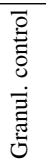 & 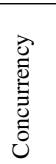 & & $\frac{\stackrel{0}{\pi}}{2}$ & $\underset{\tilde{J}}{\tilde{J}}$ & $\stackrel{0}{\stackrel{\hbar}{\hbar}}$ & 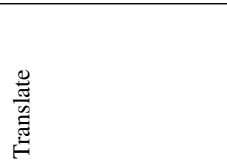 \\
\hline $\begin{array}{l}\text { ANIMALSCRIPT, } \\
\text { ANIMALSCRIPT2 }\end{array}$ & swap, add pointer & yes & yes & delay, duration & yes & no & $\begin{array}{l}\text { colors, visibility, code } \\
\text { (un)highlight }\end{array}$ & $\begin{array}{l}\text { move, move relative, } \\
\text { move along }\end{array}$ \\
\hline DsCats & insert, delete & yes & no & none & no & no & none & none \\
\hline JAWAA & $\begin{array}{l}\text { swap, push, pop, enqueue, } \\
\text { dequeue, connect nodes }\end{array}$ & yes & yes & delay & no & yes & $\begin{array}{l}\text { colors, text, width, height, font } \\
\text { size }\end{array}$ & move relative \\
\hline Matrix ASCII & none & no & no & none & no & no & none & none \\
\hline SALSA & swap, array populate & no & no & none & no & no & none & move \\
\hline Samba & none & yes & yes & delay & no & (yes) & colors, text, line width, visibility & $\begin{array}{l}\text { move/jump, } \\
\text { move/jump relative }\end{array}$ \\
\hline SVG & none & yes & yes & $\begin{array}{l}\text { delay, duration, min, } \\
\text { max, repeat, key times }\end{array}$ & yes & yes & $\begin{array}{l}\text { colors, coordinates, lengths, } \\
\text { opacity, percentages }\end{array}$ & $\begin{array}{l}\text { move, move relative, } \\
\text { move along }\end{array}$ \\
\hline
\end{tabular}

Engagement measures the support for different levels of interaction between the user and the system/language. It has two subcategories: type and level. Type gives examples of the type(s) of interaction supported by the language. Level measures the provided interaction similar to the levels of the engagement taxonomy [Naps et al. 2003]:

1. Viewing is the core of the engagement taxonomy as all the other levels of engagement include this. Viewing allows at most low level interaction with the system (e.g., moving back and forth in the animation sequence). Type can be a series of still pictures or smooth animation. It should be noted that control category corresponds to this level of the engagement taxonomy.

2. Responding requires that the language supports pausing the animation until the system gets a response from the user. Type can be, for example, a support for special purpose pop-up questions shown during the animation.

3. Changing means that the language allows interaction to be provided for modifying the algorithm visualization. For example, the input for an algorithm can be asked from the user.

4. Constructing means that the language itself is designed for writing new animations. Type determines the target of such visualizations, e.g., still pictures, algorithm animations for array manipulation, or general purpose animations.

5. Presenting means that the language can describe and supports creation of full illustrations for an audience possibly including algorithm visualizations (see constructing). Type can be general purpose slide show, hypertextbook published on the web, etc.

Evaluation The languages are evaluated in category Interaction in Table 2. Since SVG documents can include ECMAScript code and it is often used this way, we have evaluated pure SVG and SVG+ECMAScript separately. This is because the evaluation results differ significantly.

ANIMALSCRIPTs are extended to support pop-up questions during the animation, thus the languages support engagement level responding. SALSA includes commands to get input data from the user, thus it is supporting level changing. Since SVG animations can include arbitrary ECMAScript code, it can be considered to support any kind of interaction. On the other hand, pure SVG supports only viewing level. Furthermore, Matrix ASCII does not support animation, thus the none in the interaction level.
Table 2: Evaluation of the languages in category Interaction.

\begin{tabular}{|c|c|c|c|}
\hline \multirow[t]{2}{*}{ Language } & \multirow[t]{2}{*}{ Control } & \multicolumn{2}{|c|}{ Engagement } \\
\hline & & $\sum_{i}^{\infty}$ & ב্ \\
\hline $\begin{array}{l}\text { ANIMALSCRIPT, } \\
\text { ANIMALSCRIPT2 }\end{array}$ & none & $\begin{array}{l}\text { smooth animation, } \\
\text { pop-up-questions }\end{array}$ & $\begin{array}{l}\text { viewing, } \\
\text { responding }\end{array}$ \\
\hline DsCats & speed, pause & series of pictures & viewing \\
\hline JAWAA & none & smooth animation & viewing \\
\hline Matrix ASCII & none & none & none \\
\hline SALSA & none & $\begin{array}{l}\text { smooth animation, } \\
\text { user input data }\end{array}$ & $\begin{array}{l}\text { viewing, } \\
\text { changing }\end{array}$ \\
\hline Samba & none & smooth animation & viewing \\
\hline SVG & none & smooth animation & viewing \\
\hline SVG + ECMAScript & any & any & any \\
\hline
\end{tabular}

\subsection{Category Positioning}

Description Category Positioning (see Figure 5) describes the different ways to position the objects in the animation. Positioning has three subcategories: coordinates, dimensions, and layout.

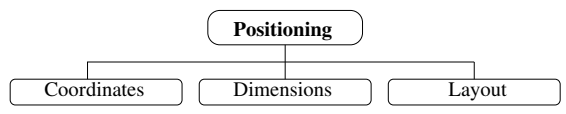

Figure 5: Category Positioning.

Coordinates list the various ways the coordinate information can be specified, and the available coordinate systems. For example, an object can be located in absolute coordinates or relative to some other location or object.

Dimensions state the maximum number of dimensions usable when positioning the objects. Usually, this value is either two or three.

Layout examines the data structure layout functionality of the language. This means, for example, how to position the nodes of a graph. The layout can be done at least in two ways: automatically or by hand. In addition, it can be a combination of these two approaches. Although automatic can be considered a feature of the system, it is also relevant for the language. For example, usage of a language that supports automatic layout of a graph differs from a language that requires the user to specify the layout providing the coordinates of the nodes in the graph. 
Evaluation The languages are evaluated in category Positioning in Table 3. DsCats, Matrix ASCII, and SALSA have only automatic layout and positioning, thus coordinates is not applicable.

Table 3: Evaluation of the languages in category Positioning.

\begin{tabular}{|l|l|l|l|}
\hline Language & Coordinates & Dimensions & Layout \\
\hline \hline $\begin{array}{l}\text { ANIMALSCRIPT, } \\
\text { ANIMALSCRIPT2 }\end{array}$ & absolute, relative to a location & 2.5 & by hand \\
\hline DsCats & $\mathrm{n} / \mathrm{a}$ & 2 & automatic \\
\hline JAWAA & absolute & 2 & by hand \\
\hline Matrix ASCII & $\mathrm{n} / \mathrm{a}$ & 2 & automatic \\
\hline SALSA & $\mathrm{n} / \mathrm{a}$ & 2 & automatic \\
\hline Samba & absolute & 2.5 & n/a \\
\hline SVG & absolute, relative & 2 & n/a \\
\hline
\end{tabular}

ANIMALSCRIPTs support depth to control how overlapping objects are drawn on the screen. Samba supports a similar feature by allowing objects to be moved backward and forward from the viewer. Thus, the dimension is 2.5 in the evaluation of these two languages.

\subsection{Category Style}

Description The category Style (see Figure 6) measures the variety of styling options available in the language. By styling, we mean setting the style of the objects in the language's vocabulary. Style is divided in five subcategories: colors, fill style, font, line style, opacity, and stylesheets.

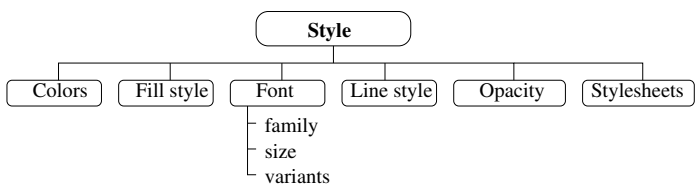

Figure 6: Category Style.

Colors is used to describe the supported color space of the language. Usually, the languages support some predefined colors and colors given as RGB-values.

Fill style states the different fill style options. These can be, for example, gradient, pattern, and solid.

Font is used to examine the variety of means to specify the typography of the text objects used. Measures of this category are family, size, and variants. Family describes the different font families usable in the language, if any. This can be, for example, Serif or monospaced. Size states if the size of the font can be changed and variants lists the different font variants available in the language. Variants can be, for example, bold or small caps.

Line style states the support for different line style options. These can be, for example, dashed, pattern, solid, and width.

Opacity states whether or not the language supports the opacity of objects. If opacity is supported it is possible to make some objects partially transparent.

Stylesheets examines the support for stylesheets in the language. Stylesheet is a style definition (i.e. colors and fonts used) defined once and applied on multiple objects. The criterion for a stylesheet support is that they have to be re-usable among objects.
Evaluation From Table 4 it can be seen that the languages are biased towards two groups: those that support styling and those that do not. DsCats, Matrix ASCII, and SALSA do not offer styling functionalities due to the automatic layout and styling, while the rest of the languages do.

The number of predefined colors and fonts available in Samba depends on the installed $\mathrm{X}$ windowing system. The fonts used in SVG document are the most flexible, and can be defined by the creator in the SVG document.

Stylesheets, opacity, and different line styles (see Table 4) are rare features in algorithm animation languages. Only ANIMALSCRIPTs and Samba have some settings for lines. Again, SVG has the most flexible styling options.

\subsection{Category Utilities}

Description The category Utilities (see Figure 7) describes the support of features that are not directly related to algorithm animation but are still useful in the animation creation process. The subcategories are comments, debug, extensible, localization, and metadata.

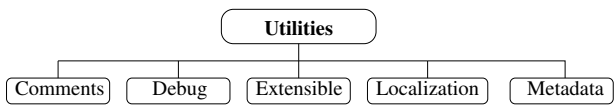

Figure 7: Category Utilities.

Subcategory comments states whether or not a language supports comments. Comments are not shown in the animation, but for the visualizer manipulating a text file.

Debug category lists the support for debugging animations. This can be, for example, printing text to the standard output.

The subcategory extensible indicates whether or not the language can be extended with reasonable effort. Such language is, for example, XML that can be extended by definition. For a language to be extensible, it must have been designed for that purpose or otherwise be easy to do. For example, a system is not considered to be extensible if it is implemented in Java and the source code is available. Yet, if there are well-defined and documented interfaces that can be implemented to add new commands to the language, the language is considered extensible.

Localization indicates whether or not the language supports localization of the animations. Usually, this is done by allowing textual data to be included in multiple languages.

Metadata describes the additional information that can be included in animations. However, it is not enough for the language to support comments that can be used to include arbitrary information. The language must have some commands or tags to describe the information as well. This metadata can be, for example, information about the author, subject, or difficulty of the animation.

Evaluation Table 5 shows the evaluation of the algorithm animation languages in category Utilities. The interesting part of the evaluation is the lack of metadata in the languages. From the algorithm animation languages evaluated, only ANIMALSCRIPTs support even a small amount of metadata. SVG, on the other hand, supports the inclusion of arbitrary metadata. ANIMALSCRIPTs' localization is an undocumented feature, thus the parenthesis. 
Table 4: Evaluation of the languages in category Style.

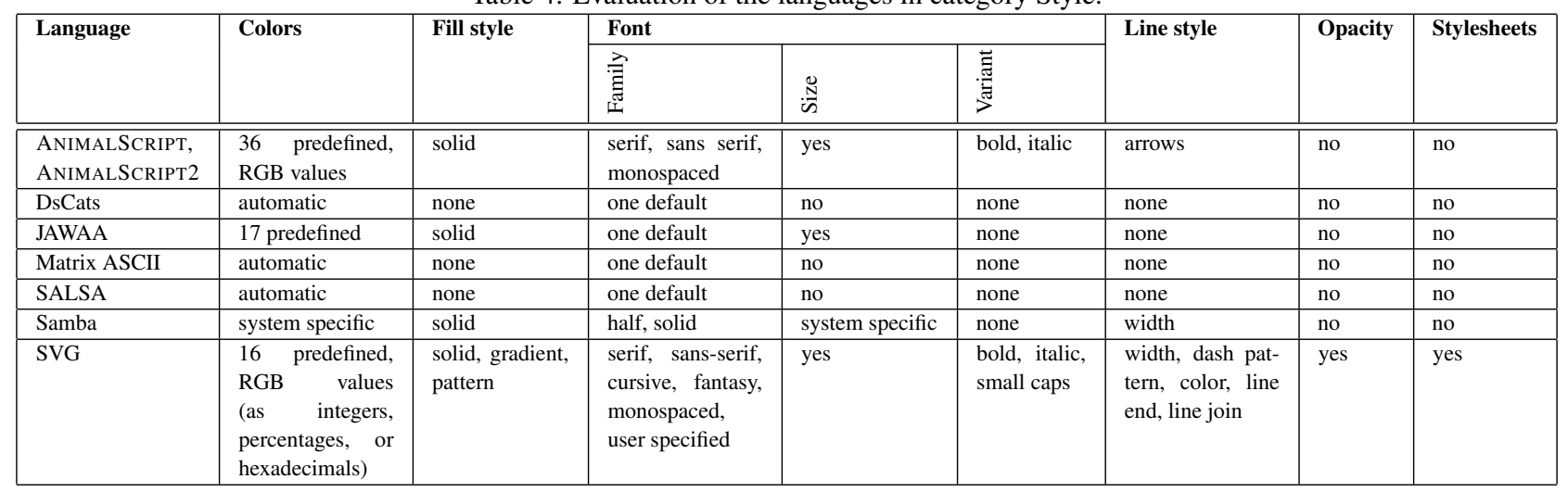

Table 5: Evaluation of the languages in category Utilities.

\begin{tabular}{|l|l|l|l|l|l|}
\hline Language & Comm. & Debug & Extens. & Local. & Metadata \\
\hline \hline $\begin{array}{l}\text { ANIMALSCRIPT, } \\
\text { ANIMALSCRIPT2 }\end{array}$ & yes & yes & yes & (yes) & author, title \\
\hline DsCats & yes & no & no & no & none \\
\hline JAWAA & no & no & no & no & none \\
\hline Matrix ASCII & yes & no & no & no & none \\
\hline SALSA & yes & no & no & no & none \\
\hline Samba & yes & yes & no & no & none \\
\hline SVG & yes & yes & yes & yes & any \\
\hline
\end{tabular}

\subsection{Category Vocabulary}

Description Category Vocabulary (represented in Figure 8) describes the amount of supported object types. Basically, these are the building blocks used to compose the animation. Vocabulary has four subcategories: data structures, graphical primitives, programming concepts, and sound.

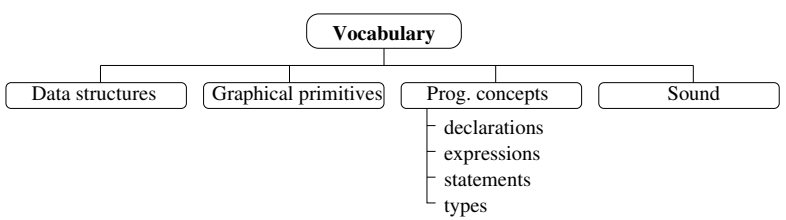

Figure 8: Category Vocabulary.

Data structures lists the different supported data structures available in the language. These can be, for example, graph, tree, and stack.

Graphical primitives lists the supported graphical primitives. Usually, there exist quite similar primitives with possibly different names. There are, however, languages where graphical primitives are not supported.

Programming concepts measure the degree of support for features similar to programming languages. There are four subcategories representing the typical constructs in programming languages: $d e c$ larations, expressions, statements, and types. The idea is not to make an exhaustive analysis of a programming language, but to give a hint on what kind of programming constructs are currently available in the animation languages. This category can be extended in the future by adopting an additional taxonomy focusing only on programming languages.

Declarations can be, for example, variable, function, or class dec- larations. Expressions can be arithmetic expressions and comparisons. Statements include iterations and assignments. Finally, types can be, for example, boolean, integer, or user defined.

Sound category states whether or not the language supports the use of sound in animations. This category is included in the taxonomy, although none of the surveyed languages supported this feature. However, we feel that sound can be a useful addition to the animations and it could be supported in algorithm animation languages as well.

Evaluation The languages are evaluated in category Vocabulary in Tables 6 and 7. Based on Table 6 it is safe to say that two types of approaches to algorithm animation exist: languages using graphical primitives and languages using data structures. However, it is good to see languages where both approaches are supported, at least to some extent.

As can be seen from Table 7, programming concepts are not that general in algorithm animation languages. However, some of the most recent languages like ANIMALSCRIPT2 and SALSA have a support for fundamental programming concepts.

Again, we have evaluated SVG and SVG+ECMAScript separately, since they have very different evaluation results. ECMAScript is a programming language, and thus it includes much more versatile programming concepts than the algorithm animation languages.

\section{Conclusion}

Many algorithm visualization tools have been developed to support teaching and learning core computer science topics such as data structures and algorithms since early 1980's. Despite the long history and the large number of systems available, no breakthrough has taken place in their widespread use in education. Several reasons for this were revealed in the survey carried out by the ITiCSE working group in 2002 [Naps et al. 2003]. Among the top five impediments identified in the survey, three are related to this work: "Time it takes to learn the new tools" ( $90 \%$ of respondents listed this), "Time it takes to develop visualizations" (90\%), and "Time it takes to adapt visualizations to teaching approach and/or course content" $(79 \%)$. All these challenges can be tackled by promoting sharing of content among algorithm animation systems.

From teacher's point of view, an ideal solution would be learning only one single visualization system that can be used to customize and adopt third party animations as well. In practice, this has been 
Table 6: Evaluation of the languages in category Vocabulary (1/2).

\begin{tabular}{|l|l|l|l|}
\hline Language & Data structures & Graphical primitives & Sound \\
\hline \hline $\begin{array}{l}\text { ANIMALSCRIPT, } \\
\text { ANIMALSCRIPT2 }\end{array}$ & array, linked list & $\begin{array}{l}\text { arc, circle, circle-segment, ellipse, point, polygons, } \\
\text { polylines, text }\end{array}$ & no \\
\hline DsCats & BST, AVL-tree, B-tree & none & no \\
\hline JAWAA & array, tree, list, graph, queue, stack & circle, line, text, rectangle, oval, polygon & no \\
\hline Matrix ASCII & array, list, graph, tree & none & no \\
\hline SALSA & array & none & no \\
\hline Samba & none & line, polygons, text, circle & no \\
\hline SVG & none & rectangles, circles, ellipses, polylines, polygons, text & no \\
\hline
\end{tabular}

Table 7: Evaluation of the languages in category Vocabulary (2/2).

\begin{tabular}{|c|c|c|c|c|}
\hline \multirow[t]{2}{*}{ Language } & \multicolumn{4}{|c|}{ Programming concepts } \\
\hline & 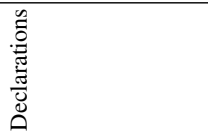 & 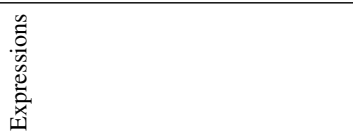 & 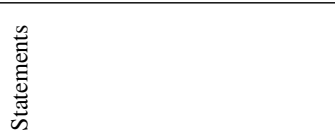 & 芯 \\
\hline ANIMALSCRIPT & none & none & none & none \\
\hline ANIMALSCRIPT2 & variables, arrays & $\begin{array}{l}+,-, *, /, \%, \|, \& \&,<,<=,== \\
=>,>, !=\end{array}$ & $\begin{array}{l}\text { conditionals, iteration, assign- } \\
\text { ment }\end{array}$ & boolean, integer, string, array \\
\hline DsCats & none & none & none & none \\
\hline JAWAA & none & none & none & none \\
\hline Matrix ASCII & none & none & none & none \\
\hline SALSA & variables, arrays & $+,-,{ }^{*}, /,<=,<,==,>,=>, !=$ & $\begin{array}{l}\text { if, while, create, set, input, pop- } \\
\text { ulate }\end{array}$ & string, number, array \\
\hline Samba & none & none & none & none \\
\hline SVG & none & none & none & none \\
\hline SVG + ECMAScript & variables, functions & $\begin{array}{l}\text { multiplicative, additive, bitwise } \\
\text { shift, relational, and equality oper- } \\
\text { ations, etc. }\end{array}$ & $\begin{array}{l}\text { block, assignment, conditionals, } \\
\text { iteration, break, continue, etc. }\end{array}$ & $\begin{array}{l}\text { boolean, number, string, ob- } \\
\text { ject, custom types }\end{array}$ \\
\hline
\end{tabular}

practically impossible due to lack of techniques to transfer animations from one visualization system to another. The ITiCSE visualization working group in 2005 [Naps et al. 2005] attacked this problem by creating a draft XML specification for algorithm animations. Such an intermediate language would support both 1) adapting visualization made by others to one's specific needs, 2) sharing animations with other people, and 3) building animation repositories. One example language partially implementing the specification is XAAL [Karavirta 2006].

In this paper, we continue to support this work by defining a taxonomy of algorithm animation languages, and applying it to evaluate a set of current languages. As a result, we have a more detailed overview of the features and properties available in them. Our evaluation could be summarized by stating that animation languages can be broadly divided into two categories: languages supporting graphical primitives and languages supporting data structures. In addition, it can be seen that SVG, especially when used together with ECMAScript, has the most advanced features in many of the categories. However, SVG is missing the data structures.

The presented taxonomy can be used in several different ways. First, it summarizes in a concise way the multitude of features available in current animation languages, and clarifies the relations among these features. Second, the taxonomy can be used as a tool for comparing different languages with each other, and understanding their strengths and limitations. Third, it provides guidelines for features needed to be taken into account in transferring animation information from one system to another. Finally, the taxonomy can be used as a reference for future development of animation systems. It can indicate what kind of features are desirable when building general purpose systems. As an example, we observed that none

\footnotetext{
${ }^{1}$ Can be achieved in Adobe SVG Viewer by using an extension.
}

of the surveyed languages support sound and even the support for controlling the interaction in the animations is rare.

Previous research has provided taxonomies (e.g., [Price et al. 1993]) for evaluating visualization systems. Our work augments this work by presenting a taxonomic evaluation of animation languages. There are many reasons why a new taxonomy is needed to make it possible to study languages and systems separately. Animation languages can provide functionality that goes beyond the basic GUI functionality covered in system taxonomies. For example, the animation language can include an extensive programming language that can be used to program the animations or the language can have properties that are not shown in the GUI such as comments and metadata. However, some systems do not have any kind of animation language even though they might provide very rich GUI functionality or the system properties are covered only partially. Thus, the taxonomy can reveal the lack of functionality in the animation language. In addition, animations created or modified in such systems cannot be transferred to other systems until there is an agreement how to support all the relevant information available in the animations. Thus, the focus is on the animation and content, not on the system properties.

We recognize that the taxonomy is not complete in the sense that it could cover all the features in all the available animation languages. Thus, as with all taxonomies, there is a constant need for evolution in the future as new important features become available. For this reason we have made an up-to-date version of the taxonomy available online $e^{2}$ hoping that others would also contribute to it.

\footnotetext{
${ }^{2}$ http://svg.cs.hut.fi/aaltaxonomy/
} 


\section{References}

Akingbade, A., Finley, T., Jackson, D., Patel, P., And RODGER, S. H. 2003. JAWAA: easy web-based animation from CS 0 to advanced CS courses. In Proceedings of the 34th SIGCSE technical symposium on Computer science education, SIGCSE'03, ACM Press, Reno, Navada, USA, 162-166.

CAppos, J., AND Homer, P., 2002. DsCats: Animating data structures for CS2 and CS3 courses. Technical paper published online. http://www.cs.arizona.edu/dscats/ dscatstechnical.pdf.

Herman, I., And Marshall, M. S. 2000. GraphXML - an XML-based graph description format. In Graph Drawing, 5262.

Hundhausen, C. D., And Douglas, S. A. 2002. Low-fidelity algorithm visualization. Journal of Visual Languages \& Computing 13, 5 (Oct.), 449-470.

KaraVirta, V., Korhonen, A., Malmi, L., AND StÅlnacke, K. 2004. MatrixPro - A tool for on-the-fly demonstration of data structures and algorithms. In Proceedings of the Third Program Visualization Workshop, Department of Computer Science, University of Warwick, UK, The University of Warwick, UK, A. Korhonen, Ed., Research Report CS-RR-407, 26-33.

Karavirta, V. 2005. XAAL - Extensible Algorithm Animation Language. Master's thesis, Department of Computer Science and Engineering, Helsinki University of Technology.

KARAVIRTA, V. 2006. Integrating algorithm animation systems. In Proceedings of the Fourth Program Visualization Workshop. To appear.

NAPS, T. L., EAGAN, J. R., AND NORTON, L. L. 2000. JHAVÉ: An environment to actively engage students in web-based algorithm visualizations. In Proceedings of the SIGCSE Session, ACM Press, New York, Austin, Texas, 109-113.

NAPs, T. L., RÖssling, G., Almstrum, V., Dann, W., FleisCher, R., Hundhausen, C., Korhonen, A., Malmi, L., McNally, M., Rodgers, S., ANd Ángel VelázquezITURBIDE, J. 2003. Exploring the role of visualization and engagement in computer science education. SIGCSE Bulletin 35, 2 (June), 131-152.

Naps, T. L., RÖSSling, G., BRUsilovsky, P., English, J., Jarc, D., Karavirta, V., Leska, C., McNally, M., Moreno, A., Ross, R. J., AND URQUiZA-Fuentes, J. 2005. Development of XML-based tools to support user interaction with algorithm visualization. SIGCSE Bulletin 37, 4 (December), 123-138.

Price, B. A., Baecker, R. M., And Small, I. S. 1993. A principled taxonomy of software visualization. Journal of Visual Languages and Computing 4, 3, 211-266.

Rössling, G., AND Freisleben, B. 2000. Program visualization using ANIMALSCRIPT. In Proceedings of the First Program Visualization Workshop, 41-52.

Rössling, G., AND Freisleben, B. 2002. ANIMAL: A system for supporting multiple roles in algorithm animation. Journal of Visual Languages and Computing 13, 3, 341-354.

Rössling, G., Gliesche, F., Jajeh, T., And Widjaja, T. 2004. Enhanced expressiveness in scripting using AnimalScript
2. In Proceedings of the Third Program Visualization Workshop, $10-17$.

STASKO, J. T. Jsamba - java version of the SAMBA animation program. Available online at http://www.cc.gatech.edu/gvu/softviz/algoanim/ jsamba/.

STASKO, J. T. 1997. Using student-built algorithm animations as learning aids. In The Proceedings of the 28th SIGCSE Technical Symposium on Computer Science Education, ACM Press, New York, San Jose, CA, USA, 25-29.

W3C, 2001. Scalable Vector Graphics (SVG) 1.0 specification. http://www.w3.org/TR/SVG, Sept.

WINTER, A. 2001. Exchanging graphs with GXL. In Graph Drawing - 9th Interational Symposium, 485-500. 